\title{
THE STRUCTURE OF TRANSLATION HALF RINGS IN EUCLIDEAN SPACES ${ }^{1}$
}

\author{
PETER H. MASERICK ${ }^{2}$
}

1. Introduction. The concept of a half ring of sets was introduced by von Neumann [2], who made extensive use of it to define and investigate measure on product spaces. A half ring $R$ is a collection of subsets of a given set satisfying the following conditions:

(i) If $R, R^{\prime} \in R$ then $R \cap R^{\prime} \in R$.

(ii) If $R, R^{\prime} \in \mathbb{R}$ and if $R \subset R^{\prime}$ then there exists a finite chain $R_{1}, R_{2}, \cdots, R_{k}, R_{i} \in \Re$ for $i=1,2, \cdots, k$ such that $R=R_{1} \subset R_{2}$ $\subset \cdots \subset R_{k}=R^{\prime}$ and $R_{j}-R_{j-1} \in R$ for $j=2,3, \cdots, k$.

The family of all bounded intervals on the real line and the family of all parallelograms in the plane with parallel sides are examples of half rings.

We shall investigate properties of half rings in $n$-dimensional $\mathrm{Er}$ clidean space $E^{n}(n \geqq 2)$. In particular, for convex nondegenerate translation half rings as defined below, we shall give a complete characterization of all such families of sets in $E^{n}$. $E^{n}$ is an $n$-dimensional Hilbert space and it will be assumed that the reader is familiar with the basic geometrical properties of such spaces, more specifically with such notions as hyperplanes, half spaces, spheres, line segments, cones, convex sets, support planes, translation of sets, etc.

A translation half ring (t.h.r.) in $E^{n}$ is a half ring $R$ of subsets of $E^{n}$ such that if $R \in R$ and if $R^{*}$ is a translate of $R$, then $R^{*} \in R$. If there exists a nondegenerate $R \in R$ (i.e. an element $R \in R$ whose interior $R^{0}$ is not empty), $R$ is a nondegenerate t.h.r.

A convex polyhedron $P$ is a subset of $E^{n}$ satisfying:

(a) $P$ is bounded,

(b) $P=\bigcap_{i=1}^{k} S_{H_{i}}$ where each $S_{H_{i}}$ is an open or closed half space with the hyperplane $H_{i}$ for its boundary.

If $P$ is nondegenerate, the intersection $H_{i} \cap \bar{P}$ of $H_{i}$ with the closure $\bar{P}$ of $P$ is defined to be a face of $P$ provided there exists a sphere $S_{e}(x)$ contained in $\bar{P}$ and tangent to $H_{i}$. The set of hyperplanes

Presented to the Society, January 28, 1958 under the title Set functions and translation half-rings in the plane; received by the editors December 6, 1957 and, in revised form, June 21, 1958.

1 This research was supported by the United States Air Force through the Air Force Office of Scientific Research and Development Command, and contract No. AF 18(603)-78.

2 The author wishes to express his appreciation to Professor R. E. Fullerton for his interest and assistance. 
which determine faces of a nondegenerate polyhedron $P$ also determines a minimal collection of half spaces whose intersection is $P$ and which is contained in any other collection of half spaces which determine $P$. It also follows that $H_{i} \cap \bar{P}$ is a face of $P$ if and only if there exists $x \in H_{i} \cap \bar{P}$ and a sphere $S_{\epsilon}(x)$ with center at $x$ such that the disk $H_{i} \cap S_{\epsilon}(x)$ is a subset of $H_{i} \cap \bar{P}$. If $P$ is degenerate, this can be used as the definition of a face of $P$. The faces $H_{i} \cap \bar{P}$ of $P$ then generate unique hyperplanes.

If a family of hyperplanes consists of parallel hyperplanes, they are said to have the same direction $d$. A collection $\beta$ of convex polyhedra is oriented with respect to a set $D$ of directions if every $P \in P$ has only faces which generate hyperplanes of directions $d \in D$. The faces are said to have the direction of the respective hyperplanes they generate.

\section{The structure of half rings in $E^{n}$.}

THEOREM 1. The set of all convex polyhedra forms a half ring in $E^{n}$.

Proof. The intersection of two convex polyhedra is again a convex polyhedron.

To prove the second half ring axiom let $P, P^{\prime} \in P$ and $P \subset P^{\prime} . P$ can be written as $\bigcap_{i=1}^{k-1} S_{H_{i}}$ where each $H_{i} \cap \bar{P}=F_{i}$ is a face of $P$. Let $P_{1}=P, P_{i+1}=P_{i} \cup F_{i}$ for $i=1,2, \cdots, k-1$. Clearly $P_{k}=\bar{P}$ and $\left\{P_{i}\right\}_{i=1,2, \ldots, k}$ is a chain extension from $P$ to $\bar{P}$ which satisfies (ii). Thus without loss of generality we can assume $P=\bar{P}$. Therefore $P$ can be expressed as $\bigcap_{i=1}^{k-1} \bar{S}_{H_{i}}$. Let $P_{1}=P, P_{j}=\left(\bigcap_{i=j}^{k-1} \bar{S}_{H_{i}}\right) \cap P^{\prime}$ for $j \leqq k-1$ and $P_{k}=P^{\prime}$. Each $P_{j}$ is a convex polyhedron and $P_{j} \supset P_{j-1}$ for $j=2,3, \cdots, k$. If $2 \leqq j \leqq k-1$ then

$$
\begin{aligned}
P_{j} & -P_{j-1}=\left(\bigcap_{i=j}^{k-1} \bar{S}_{H_{i}}\right) \cap P^{\prime}-\left(\bigcap_{i=j-1}^{k-1} \bar{S}_{H_{i}}\right) \cap P^{\prime} \\
& =\left(\bigcap_{i=j}^{k-1} \bar{S}_{H_{i}}-\bigcap_{i=j-1}^{k-1} \bar{S}_{H_{i}}\right) \cap P^{\prime}=\left(E^{n}-\bar{S}_{H_{j-1}}\right) \cap\left(\bigcap_{i=j}^{k-1} \bar{S}_{H_{i}}\right) \cap P^{\prime} .
\end{aligned}
$$

But $\left(E^{n}-\bar{S}_{H_{j-1}}\right)$ is an open half space and so $P_{j}-P_{j-1} \in \odot$. If $j=k$ similar reasoning will show that $P_{k}-P_{k-1} \in \mathcal{P}$. Therefore $\left\{P_{i}\right\}_{i=1,2, \cdots, k}$ is the desired chain extension from $P_{1}=P$ to $P_{k}=P^{\prime}$.

COROLlaRy. If $\odot$ is the set of all convex polyhedra oriented with respect to a given set of directions then $P$ is a half ring.

LEMMA. If $P$ is a half ring of convex polyhedra, $P, P^{\prime} \in P$ such that $P \subset P^{\prime},\left\{P_{i}\right\}_{i=1,2, \cdots, k}$ is the finite chain of (ii) and if $P_{i-1}$ is nondegenerate then $P_{i-1}=S_{H} \cap P_{i}$ where $H \cap \bar{P}_{i-1}$ is some face of $P_{i-1}$. 
Proof. Since $P_{i-1} \subset P_{i}$ we can write $P_{i-1}=\left(\bigcap_{j=1}^{l} S_{H_{j}}\right) \cap P_{i}$. Assume that each $\left(\bar{P}_{i-1} \cap H_{j}\right) \cap P_{i}^{0}$ contains a point $x_{j}$ such that $x_{j} \in U_{t \neq j} H_{t}$. If $l>1$ then the line segment $\left[x_{1}, x_{2}\right]$ contains an interior point $z$ of $P_{i-1}$. For if $\left[x_{1}, x_{2}\right] \subset b\left(P_{i-1}\right)$, since $\left[x_{1}, x_{2}\right] \subset P_{i}^{0}$ then for each $z \in\left[x_{1}, x_{2}\right]$ there must be an $H_{j}$ for $j=1,2, \cdots, l$ such that $z \in H_{j}$. But there are infinitely many points on $\left[x_{1}, x_{2}\right]$ and hence some $H_{j}$ must contain at least two distinct points. But if a hyperplane contains two points of a line it contains the entire line. Thus we reach the contradiction that $x_{1}, x_{2} \in H_{j}$ for some $j$.

Having shown the existence of $z \in\left[x_{1}, x_{2}\right]$ such that $z \in P_{i-1}^{0}$ we note that since $x_{1}, x_{2} \in b\left(P_{i-1}\right) \cap P_{i}^{0}$ then $x_{1}, x_{2} \in b\left(P_{i}-P_{i-1}\right)$. But then by convexity we reach the contradiction that $z \in\left[x_{1}, x_{2}\right] \subset b\left(P_{i}-P_{i-1}\right)$ or $z \in\left(P_{i}-P_{i-1}\right) \cap P_{i-1}^{0}$. Thus $l=1$.

TheOREM 2. If $P$ is a nondegenerate t.h.r. of convex polyhedra, then for every finite set $\left\{d_{i}\right\}_{i=1,2, \cdots, m}$ of distinct directions of $\mathcal{P}, \mathcal{P}$ contains a nondegenerate polyhedron $R$ with exactly two faces of each such direction.

Proof. (a). It will be shown that for each direction $d$ of $P$, there exists a nondegenerate member of $\beta$ with direction $d$.

If $P$ is degenerate and has direction $d$, since $P$ is nondegenerate there exists $P^{\prime} \in \mathcal{P}, z \in E^{n}$ such that $z \in\left(P^{\prime}\right)^{0}$. Translate $P$ into $P^{*}$ such that $z \in P^{*}$. $P^{*} \cap P^{\prime}=P_{1}$ can be extended into $P^{\prime}$ by a finite chain $\left\{P_{i}\right\}_{i=1,2, \cdots, k}$ of (ii). If $P_{2}$ is nondegenerate and does not have direction $d$ then $P_{1} \cap P_{2}^{0} \neq \varnothing$ ( $\varnothing$ is used to denote the null set). But since $P_{1}$ is degenerate, we reach the contradiction that $P_{2}-P_{1}$ is not convex. If $P_{2}$ is degenerate then by repeatedly applying the above argument we can produce a nondegenerate $P_{i}$ with direction $d$.

(b) Let $P$ be a nondegenerate member of $P$ with a face $H \cap \bar{P}$ of direction $d$. Since $P$ is a t.h.r. we can assume the existence of a nondegenerate $P^{\prime}$ such that $\left(P^{\prime}\right)^{0} \supset H \cap \bar{P}$. Let $\left\{P_{i}\right\}_{i=1,2, \cdots, k}$ be the chain extension from $P$ to $P^{\prime}$. By the lemma there exists $i<k$ such that $P_{i-1}=S_{H} \cap P_{i}$ where $H \cap \bar{P}_{i-1}$ is a face of $P_{i-1}$. If we take the largest such $i$ it is apparent that $P_{i}-P_{i-1}$ is nondegenerate. Thus $P_{i-1}$ and $P_{i}-P_{i-1}$ are nondegenerate members of $P$ such that each of them have direction $d$ and the intersection of their determining half spaces of these directions is not a half space no matter how these polyhedra are translated.

(c) Let $\left\{P_{i j}\right\}_{\substack{j=1,2 \\=1,2}, m}$ be a collection of polyhedra such that each $P_{i j}$ has direction $d_{i}$ with corresponding half spaces $S_{H_{i j}}$ where $S_{H_{i 1}} \cap S_{H_{i 2}}$ is not a half space. There exists $\epsilon>0$ and $x_{i j} \in P_{i j}$ such that $S_{\epsilon}\left(x_{i j}\right) \subset P_{i j}$ and $S_{\epsilon}\left(x_{i j}\right)$ is tangent to $P_{i j}$ at $H_{i j}$. Since $\odot$ is a 
t.h.r. we can assume that the $x_{i j}$ 's coincide. From the definitions of a face of a polyhedron and direction stated in the introduction, it is clear that $\bigcap_{i=1 ; j=1,2}^{m} P_{i j}$ is the desired polyhedron.

If $P$ is a half ring of convex polyhedra oriented with respect to $m$ distinct directions then since no convex polyhedra can have more than two faces of any given direction, it follows that no $P \in \mathcal{P}$ can have more than $2 m$ faces. Examples can be constructed to show that not every half ring $P$ of convex polyhedra with an upper bound on the number of faces is oriented with respect to a finite number of directions. However, as the following corollary shows, this cannot occur in a nondegenerate t.h.r.

Corollary 1. A nondegenerate t.h.r. $\odot$ of convex polyedra is oriented with respect to a finite number of directions if and only if there exists a number $m$ such that each $P \in \mathcal{P}$ has fewer than $m$ faces.

Proof. The proof follows immediately from the foregoing statement and Theorem 2.

A nondegenerate polyhedron $R \subset E^{n}$ is a parallelepiped if $R$ has exactly $n$ distinct directions. It is known that a nondegenerate parallelepiped has exactly $2 n$ faces. It is further known that every nondegenerate polyhedron has at least $n$ distinct directions and hence a nondegenerate parallelepiped $R$, oriented with respect to these $n$ directions exists. Moreover, $R$ can be determined so that it will be contained within any arbitrarily small sphere. If a parallelepiped can be oriented with respect to $n$ directions, these directions are said to be independent.

COROLlaRY 2. If $\odot$ is a nondegenerate t. h. r. in $E^{n}$ and if $\left\{d_{i}\right\}_{i=1,2, \cdots, n}$ is any set of $n$ independent directions of $\odot$, then there exists in $\odot$ a nondegenerate parallelepiped $R$ with these $n$ directions.

Proof. Let $\left\{P_{i j}\right\}_{i=1,2}^{j-1,2} \ldots, n$ be a collection of elements of $P$ constructed as in part (b) of the proof of Theorem 2 and described in part (c) of the same proof $\left(P_{i j}\right.$ has direction $\left.d_{i j}\right)$. Let $R$ be a parallelepiped oriented with respect to $\left\{d_{i}\right\}_{i=1,2, \ldots, n}$ such that for each $P_{i j}, R$ can be translated into $R_{i j}$ where $R_{i j}$ will be contained in $P_{i j}$ with its face of direction $d_{i}$ in a corresponding face of $P_{i j}$. Translate each $P_{i j}$ into $P_{v}^{*}$ so that the corresponding translates $R_{i j}^{*}$ of $R_{i j}$ will coincide. It is then clear that $\bigcap_{i=1 ; j=1,2}^{n} P_{i j}$ will be the desired parallelepiped.

Corollary 3. The set $R$ of all parallelepipeds and degenerate polyhedra with respect to $n$ directions is a half ring.

Proof. From Theorem 1 the set $\odot$ of all polyhedra oriented with 
respect to these $n$ directions is a half ring. But each nondegenerate $P \in \mathcal{P}$ must have at least $n$ directions to be bounded. Therefore $P=R$.

COROLlaRY 4. $\odot$ is a nondegenerate t.h.r. of parallelepipeds and degenerate polyhedra oriented with respect to $n$ distinct directions if and only if each nondegenerate $P \in P$ has the same number of faces.

Proof. By Corollary 1, $\rho$ is oriented with respect to a finite number $k$ of directions. By Theorem 2, there exists $P \in P$ with at least $2 k$ and hence exactly $2 k$ faces. But by Corollary 2 there is $R \in \mathcal{P}$ with $n$ directions and $2 n$ faces. Therefore $2 k=2 n$ or $n=k$ and hence $\beta$ is a set of parallelepipeds and degenerate polyhedra oriented with respect to $n$ distinct directions.

\section{The characterization of nondegenerate convex translation half rings.}

THEOREM 3. If $\mathcal{G}$ is a t.h.r. of bounded convex subsets of $E^{n}$ then the closure $\bar{G}$ of each $G \in \mathcal{G}$ is a convex polyhedron.

The proof of Theorem 3 will depend on the five lemmas included below. A boundary point $x$ of a convex subset $G$ is a support point if there is at most one support tangential hyperplane $H$ containing $x$. It is known [1] that for each boundary point $y(y \in b(G))$ there is at least one support hyperplane $H_{y}$ containing $y$.

Lemma 1. If the boundary point $x$ of a convex set $G$ is a boundary point for a sphere $S_{\epsilon}(y) \subset G$ then $x$ is a support point for $G$.

Proof. It can be shown from Hilbert space considerations that every point of a sphere is a support point for the sphere. Therefore there is at most one hyperplane $H_{x}$ supporting $S_{c}(y)$ at $x$. However there exists a hyperplane $H_{x}^{\prime}$ which is supporting for $G$ at $x$ and hence for $S_{\epsilon}(y)$ at $x$. Thus $H_{x}^{\prime}=H_{x}$.

Lemma 2. If $G$ is nondegenerate and convex and if $x \in b(G)$ then $\bar{S}_{\epsilon}(x)$ contains a support point of $G$ for every $\epsilon>0$.

Proof. The boundary $b(G)$ is closed and every closed sphere in $E^{n}$ is compact. Since $x \in b(G)$ there exists $y \in S_{\epsilon / 2}(x) \cap G^{0}$. Since $b(G)$ is closed, $b(G) \cap \bar{S}_{\epsilon}(x)$ is compact. Therefore the distance $\delta$ from $y$ to $\bar{S}_{\epsilon}(x) \cap b(G)$ is assumed, say at $z \in \bar{S}_{\epsilon}(x) \cap b(G)$. Since the distance from $x$ to $y$ is less than $\epsilon / 2, \delta<\epsilon / 2$. But if $u \in b(G)$ and $u \notin \bar{S}_{\epsilon}(x)$ the distance from $u$ to $y$ is larger than $\epsilon / 2$. Thus $S_{\delta}(y)$ is tangent to $b(G)$ at and only at points of $\bar{S}_{\epsilon}(y) \cap b(G)$ and hence $S_{\delta}(y) \subset G$. Therefore by Lemma $1, S_{\epsilon}(x)$ must contain a support point of $G$. 
Lemma 3. If $G$ is closed, nondegenerate, convex and if $\left\{S_{H_{x}}\right\}$ is the collection of all support half spaces containing support points $x$ then $\cap S_{H_{x}}=G$.

Proof. Clearly $\cap S_{H_{x}} \supset G$. If $z \in \cap S_{H_{x}}$ and $z \notin G$ then every line segment from $z$ to an interior point of $G$ contains a boundary point of $G$. Therefore the cone $C$ determined by $z$ and an interior sphere $S_{\delta}(v)$ of $G$ contains a point $y \in b(G)$ on its axis. Hence there exists $\bar{S}_{\epsilon}(y) \subset C$. $\bar{S}_{\epsilon}(y) \cap b(G)$ contains no support point of $G$. For if $w$ were such a point then since the support half space $S_{H_{v}} \supset G \supset S_{\delta}(v)$ and since there is a $u \in S_{\delta}(v)$ such that $w \in[z, u] \cap b(G)$ we have the contradiction that $z \notin S_{H_{w}}$. Hence $\bar{S}_{\epsilon}(y) \cap b(G)$ does not contain a support point which contradicts Lemma 2. So $z \in G$ and thus $\cap S_{H_{x}}=G$.

Lemma 4. If $x$ and $y$ are support points of a convex bounded set $G$ such that $x$ and $y$ determine distinct support hyperplanes, then the line segment $(x, y)$ (not including its endpoints) is contained in $G^{0}$.

Proof. If $(x, y) \nsubseteq G^{0}$ then $z=x / 2+y / 2 \in b(G)$. Thus $z$ can be covered by a support hyperplane $H_{z}$. If $H_{z}$ contains either $x$ or $y$ then it contains $x$ and $y$. Therefore from hypothesis $H_{z}$ contains neither $x$ nor $y$. But $x \in b(G)$ and so $x \in S_{H_{s}}$. But then $y \notin S_{H_{s}}$ and this contradicts the fact that $y \in b(G)$.

Lemma 5. If $G$ is a nondegenerate convex set but not a polyhedron then $b(G)$ contains an infinite sequence $\left\{x_{n}\right\}$ of distinct points such that the open segment $\left(x_{i}, x_{j}\right) \subset G^{0}$ for $i \neq j$.

Proof. If $G$ is not a polyhedron then there exists an infinite number of support points (Lemma 3 ) each determining unique support hyperplanes. By Lemma $4\left(x_{i}, x_{j}\right) \subset G^{0}$ for $i \neq j$.

Proof of THEOREM 3. (a) If $G \in \mathcal{G}$ is nondegenerate but not a convex polyhedron there exists an infinite sequence of points of $b(G)$ satisfying the conclusion of Lemma 5 . Since $\bar{G}$ is bounded, this sequence is bounded and therefore has a subsequence $\left\{x_{i}\right\}$ which converges to some point $x_{0} \in b(\bar{G})$. Thus every sphere $S_{\epsilon}\left(x_{0}\right)$ with center at $x_{0}$ contains a convergent subsequence $\left\{x_{i j}\right\}$ of $\left\{x_{i}\right\}$.

Translate $G$ into $G^{*}$ such that $x_{0}^{*} \in G^{0}$. The collection $\left\{G_{t}-G_{t-1}, G_{1}\right\}_{t=2,3, \cdots, k}$, where each $G_{t}$ is a member of the finite chain from $G_{1}=G^{*} \cap G$ to $G$, is a partition of $G$. Each translate $x_{i_{j}}^{*}$ of $x_{i j}$ is such that $x_{i_{j}}^{*} \in b\left(G_{t}-G_{t-1}\right)$ for some $t$. Since the sequence $\left\{x_{i_{j}}^{*}\right\}$ is infinite and $\left\{G_{l}-G_{t-1}\right\}$ is finite, there exists $m \geqq 2$ and $z_{1}, z_{2} \in\left\{x_{i_{j}}^{*}\right\}$ such that $z_{1}, z_{2} \in b\left(G_{m}-G_{m-1}\right)$. But since $\left(z_{1}, z_{2}\right) \subset G_{1}^{0}$, $\left(z_{1}, z_{2}\right) \mathscr{C}\left(G_{m}-G_{m-1}\right)$. But then $\left(z_{1}, z_{2}\right)$ must be contained in the closure 
of $G_{m}-G_{m-1}$ and hence we reach the contradiction that this closure contains an interior point of $G_{1}$.

(b) If $G$ is degenerate then unless $G$ is either a line segment or a point, in which case $G$ is a convex polyhedron, there exists $k<n$ such that $G$ is a nondegenerate subset of $E^{k}$. Part (a) is then a valid argument showing that $G$ is a polyhedron in $E^{k}$ and hence $G$ is a degenerate polyhedron in $E^{n}$.

\section{REFERENCES}

1. V. L. Klee, Jr., Convex sets in linear spaces, Duke Math. J. vol. 18 (1951) pp. $443-465$. 1950.

2. J. von Neumann, Functional operators, Princeton University Press, Princeton,

UNIVERSITY OF MARYLAND 